CERN-TH.6221/91

\title{
$Z$ Boson Decay into a Fermion-Antifermion Pair and an Arbitrary Number of Hard Photons
}

\author{
W. J. Stirling ${ }^{1}$ \\ Theory Division, CERN, \\ Geneva, Switzerland
}

\begin{abstract}
The partial widths for the decay of a $Z$ boson into a massless fermion pair and an arbitrary number of hard acollinear photons, $\mathrm{J}(Z \rightarrow f \vec{f}+n \gamma)$, are calculated. Such decays are an important background to a variety of new physics processes involving new particles decaying into photons and light fermions. The appropriate matrix elements are calculated exactly using 'spinor techniques'. Some sample numerical results are presented for $n_{\gamma} \leq 4$.
\end{abstract}

CERN-TH.6221/91

August 1991

\footnotetext{
${ }^{1}$ Permanent Address: Department of Physics, University of Durham, Durham, England.
} 


\section{Introduction}

QED radiative corrections to the basic $e^{+} e^{-} \rightarrow Z \rightarrow f \bar{f}$ processes at LEP and SLC are of fundamental importance in deriving tests of the Standard Model. Techniques, both Monte Carlo and analytic, have been developed for analysing the emission of an arbitrary number of soft and collinear photons emitted from the initial and final state fermions [1]. These have an important influence on, for example, the $Z$ line shape, the forward-backward asymmetries, small-angle Bhabha scattering etc. In the absence of cuts, these multiphoton soft and collinear configurations do indeed dominate the cross section. However at a much smaller rate energetic, well-separated ('hard') photons can also be emitted. For such processes, soft and collinear leading-logarithm approximations - as implemented for example in Monte Carlos and exponential form factors - are clearly no longer appropriate, and the correct theoretical tools are exact matrix elements. Processes involving the emission of hard photons are important not so much as a test of QED, but rather because they are backgrounds to possible new physics processes. For example, the decay $Z \rightarrow Z^{*}(\rightarrow f \bar{f}) X(\rightarrow \gamma \gamma)$, where $X$ is a new massive state (for example, the Standard Model Higgs boson), would give rise to a final state with a fermion-antifermion pair and two well-separated photons. The same is true for the pair production and radiative decay of 'excited fermions': $Z \rightarrow f^{*} \bar{f}^{*} \rightarrow f \bar{f} \gamma \gamma$.

In this paper we calculate the matrix elements for $Z \rightarrow f \bar{f}+n \gamma$. By normalizing to the leading order $Z \rightarrow f \bar{f}$ decay we obtain the probabilities for the emission of an arbitrary number of hard photons. Obviously each photon emission 'costs' a power of $\alpha \ll 1$, and so in practice we restrict our numerical estimates to $n \leq 4$. With $(n+1)$ ! Feynman diagrams for a $n$-photon process, the calculation is only tractable using 'spinor techniques', where the complex amplitudes are calculated numerically for each spin/helicity configuration $[2,3]$. The method we use is in fact very similar to that for the calculation of $e^{+} e^{-} \rightarrow n \gamma$ presented in reference [4]. Compact expressions for the matrix elements are presented in the next section. In section 3, we illustrate our results by calculating the decay probabilities for the case when the photons are kept separate from the fermions by an invariant mass cut. We consider both $\mu^{+} \mu^{-}$ and hadronic (i.e. $\sum q \bar{q}$ ) final states.

Note that the one-photon calculation is trivial and can be performed analytically. Exact two-photon bremsstrahlung corrections to $e^{+} e^{-} \rightarrow f \vec{f}$ were first calculated in reference [5], from which the final-state photon emission contributions on the $Z$ pole could in principle be extracted.

\section{Calculation of the matrix elements}

The calculation of the partial decay width $\Gamma(Z \rightarrow f \bar{f}+n \gamma)$ is performed using the 'spinor techniques' described in reference [2], which should be consulted for more 
details about the definition of the fundamental spinors, polarization vectors, etc. Here we simply list the result.

Although we are interested in the partial decay width of an on-shell $Z$ boson, the quantity we actually calculate is the peak cross cross section, i.e. $\sigma\left(e^{+} e^{-} \rightarrow Z \rightarrow\right.$ $f \bar{f}+n \gamma)$ with $\sqrt{s}=M_{Z}$. This allows us to correctly include the correlations between the initial and final state particle momenta, as manifest for example in the forwardbackward lepton asymmetry. The peak cross section is trivially related to the decay width by

$$
\left.\sigma\left(e^{+} e^{-} \rightarrow Z \rightarrow X\right)\right|_{\sqrt{s}=M_{Z}}=\frac{12 \pi \Gamma_{e e} \Gamma(Z \rightarrow X)}{M_{Z}^{2} \Gamma_{t o t}^{2}} .
$$

By restricting ourselves to the peak cross section we can neglect the effects of initial state radiation and $s$-channel photon- $Z$ interference. We can assume that in practice additional cuts will be applied to the final state particles to keep them well-separated from the beam direction. Of course the actual value of the peak cross section is very sensitive to electroweak radiative corrections, but these will tend to cancel when we form the ratios of the $n$-photon to zero-photon cross sections. We set all fermion masses to zero, which is certainly adequate for final states where all pairs of invariant masses are required to be large. The massive fermion calculation, although more complicated, could be performed using the generalization of massless-fermion spinor techniques described in reference [4].

The momentum and helicity labelling is

$$
e^{-}\left(p_{1}, \nu_{1}\right)+e^{+}\left(p_{2}, \nu_{2}\right) \rightarrow f\left(p_{3}, \nu_{3}\right)+\vec{f}\left(p_{4}, \nu_{4}\right)+\gamma\left(k_{1}, \lambda_{1}\right)+\ldots+\gamma\left(k_{n}, \lambda_{n}\right),
$$

and because of helicity conservation at the fermion - gauge-boson vertices, the independent helicity labels can be chosen to be $\nu_{1}, \nu_{3}$ and the $\lambda_{i}$.

The $n$-photon cross section has the generic form

$$
\sigma_{n}=\frac{1}{2 M_{Z}^{2} n !} \int d \Phi_{n} \frac{1}{4} \sum_{\nu_{1}, \nu_{3}, \lambda_{i}= \pm}\left|\mathcal{M}_{n}\right|^{2}
$$

with

$$
\begin{aligned}
\sum_{\nu_{1}, \nu_{3}, \lambda_{i}= \pm}\left|\mathcal{M}_{n}\right|^{2}= & \frac{g_{Z}^{4} e_{f}^{2 n}}{M_{Z}^{2} \Gamma_{\text {tot }}^{2}} \sum_{\lambda_{i}= \pm}\left[\left(v_{e}-a_{e}\right)^{2}\left(v_{f}-a_{f}\right)^{2}\left|Z_{n}\left(k_{i}, \lambda_{i} ; p_{1}, p_{2}, p_{3}, p_{4}\right)\right|^{2}\right. \\
& +\left(v_{e}+a_{e}\right)^{2}\left(v_{f}-a_{f}\right)^{2}\left|Z_{n}\left(k_{i}, \lambda_{i} ; p_{2}, p_{1}, p_{3}, p_{4}\right)\right|^{2} \\
& +\left(v_{e}-a_{e}\right)^{2}\left(v_{f}+a_{f}\right)^{2}\left|Z_{n}\left(k_{i}, \lambda_{i} ; p_{1}, p_{2}, p_{4}, p_{3}\right)\right|^{2} \\
& \left.+\left(v_{e}+a_{e}\right)^{2}\left(v_{f}+a_{f}\right)^{2}\left|Z_{n}\left(k_{i}, \lambda_{i} ; p_{2}, p_{1}, p_{4}, p_{3}\right)\right|^{2}\right]
\end{aligned}
$$

Note that $g_{Z}^{2}=\sqrt{2} G_{F} M_{Z}^{2}$ and $a_{e}=-1 / 2, v_{e}=-1 / 2+2 \sin ^{2} \theta_{W}$ etc. The four terms in the above are the amplitudes for $\nu_{1}, \nu_{3}= \pm$.

The complex scattering amplitude $Z_{n}$ corresponds to definite helicity incoming and outgoing fermions, and arbitrary helicity photons. The $(n+1)$ ! Feynman diagrams 
for $Z_{n}$ are simply the $n$ ! permutations of the photons attached to the final state fermion line, with $n+1$ ways of partitioning the photons on either side of the $Z f \bar{f}$ vertex, i.e.

$$
Z_{n}\left(k_{i}, \lambda_{i} ; p_{1}, p_{2}, p_{3}, p_{4}\right)=\sum_{\text {perms. }\{k, \lambda\}=\{q, \kappa\}} \sum_{m=0}^{n} X\left(m, n-m ; q_{i}, \kappa_{i} ; p_{1}, p_{2}, p_{3}, p_{4}\right) .
$$

The amplitude $X$ contains spinor products and propagators:

$$
X\left(m, n-m ; q_{i}, \kappa_{i} ; p_{1}, p_{2}, p_{3}, p_{4}\right)=(-1)^{n-m} \prod_{i=1, n} \frac{1}{\sqrt{p_{0} \cdot q_{i}}} D_{3} D_{4} Y_{3} Y_{4},
$$

where

$$
\begin{aligned}
D_{3}= & \prod_{i=1}^{m}\left[\left(p_{3}+\sum_{j=1, i} q_{j}\right)^{2}\right]^{-1} \\
D_{4}= & \prod_{i=1}^{n-m}\left[\left(p_{4}+\sum_{j=1}^{i} q_{n+1-j}\right)^{2}\right]^{-1} \\
Y_{3}= & s\left(p_{3}, b_{1}\right) \prod_{i=1}^{m-1}\left[t\left(a_{i}, p_{3}\right) s\left(p_{3}, b_{i+1}\right)+\sum_{j=1}^{i} t\left(a_{i}, q_{j}\right) s\left(q_{j}, b_{i+1}\right)\right] \\
& \times\left[t\left(a_{n}, p_{3}\right) s\left(p_{3}, p_{1}\right)+\sum_{j=1}^{m} t\left(a_{m}, q_{j}\right) s\left(q_{j}, p_{1}\right)\right] \\
Y_{4}= & t\left(a_{n}, p_{4}\right) \prod_{i=1}^{n-m-1}\left[t\left(a_{n-i}, p_{4}\right) s\left(p_{4}, b_{n+1-i}\right)+\sum_{j=1}^{i} t\left(a_{n-i}, q_{n+1-j}\right) s\left(q_{n+1-j}, b_{n+1-i}\right)\right] \\
& \times\left[t\left(p_{2}, p_{4}\right) s\left(p_{4}, b_{m+1}\right)+\sum_{j=1}^{n-m} t\left(p_{2}, q_{n+1-j}\right) s\left(q_{n+1-j}, b_{m+1}\right)\right] .
\end{aligned}
$$

The massless four-vectors $a_{i}, b_{i}$ are defined as follows:

$$
\begin{array}{ll}
\kappa_{i}=+: & a_{i}=p_{0}, b_{i}=q_{i} \\
\kappa_{i}=-: & a_{i}=q_{i}, b_{i}=p_{0} .
\end{array}
$$

The four-vector $p_{0}^{\mu}$ is an arbitrary, massless momentum vector which specifies the photon gauge. The matrix-element squared is independent of $p_{0}$, and any choice not parallel to the $q_{i}$ is acceptable. The $s$ and $t$ spinor products are defined by [2]

$$
\begin{aligned}
s\left(p_{i}, p_{j}\right) & =\left(p_{i}^{y}+i p_{i}^{z}\right)\left(\frac{p_{j}^{0}-p_{j}^{x}}{p_{i}^{0}-p_{i}^{x}}\right)^{\frac{1}{2}}-\left(p_{j}^{y}+i p_{j}^{z}\right)\left(\frac{p_{i}^{0}-p_{i}^{x}}{p_{j}^{0}-p_{j}^{x}}\right)^{\frac{1}{2}} \\
t\left(p_{i}, p_{j}\right) & =-s^{*}\left(p_{i}, p_{j}\right)
\end{aligned}
$$

for any pair of massless four-momenta appearing in the problem. There is some arbitrariness in the definition of $s$ and $t$, but the above - with $z$ labelling the beam 
direction and $x$ and $y$ perpendicular to it - is convenient in practice. The above expression for the cross section, although at first sight unwieldy, is in fact not difficult to program. The most attractive feature is that the same general expression is valid for any number of photons.

For the phase space integration we use the RAMBO event generator [6], which returns constant phase-space weights for massless multiparticle final states. Since we use photon cuts which deliberately avoid phase-space regions where the matrix elements are singular, the overall efficiency of the numerical calculation is quite satisfactory. ${ }^{2}$ Several cross-checks on the calculation have been performed: (i) gauge invariance, (ii) comparison with the analytic $(n=1)$ result, and (iii) comparison with a QED version of an existing 'four-jet' program [7] $(n=2)$.

\section{Phenomenology}

In practice, the exact cuts on the fermions and photons will be determined by the nature of the expected signal for which the present analysis provides a background. However, to illustrate the overall size of the decay rates it is sufficient to use a simple set of minimal cuts on the photons only. The typical cuts appropriate for this type of calculation are photon energy and photon-fermion angle cuts. These can be implemented simultaneously by using a 'JADE algorithm' type invariant-mass cut [8]. Thus we introduce a dimensionless parameter $y_{\text {cut }}$, and require that the invariant mass of each photon-fermion pair be larger than $\sqrt{y_{\text {cut }}} M_{Z}$, i.e.

$$
\begin{aligned}
& \left(p_{3}+k_{i}\right)^{2}>y_{\text {cut }} M_{Z}^{2} \\
& \left(p_{4}+k_{i}\right)^{2}>y_{\text {cut }} M_{Z}^{2},
\end{aligned}
$$

where the $k_{i}^{\mu}$ are the four-momenta of the photons and $p_{3}^{\mu}, p_{4}^{\mu}$ are the momenta of the final-state (massless) fermion, antifermion respectively. Note that the single parameter $y_{\text {cut }}$ protects the matrix elements from the soft and collinear singular configurations. The maximum value of $y_{\text {cut }}$ for a non-zero decay rate depends on the number of photons:

$$
y_{\text {cut }}^{\max }(n)=\frac{1}{2 n} .
$$

Note that we do not impose any isolation cut between the photons themselves we assume that the photon-photon resolution is in practice much smaller than the $y_{\text {cut }}$ separation of the photons and fermions. To begin with, we also do not impose any separation cut between the fermion and antifermion, as would be appropriate, for example, for $f=\mu$. For this reason the one-photon region corresponds to $0 \leq$ $y_{\text {cut }} \leq 1 / 2$, compared to the upper limit of $1 / 3$ familiar from three-jet analyses. Configurations close to the upper limits defined in eq.(11) correspond to collinear

\footnotetext{
${ }^{2}$ The self-contained FORTRAN program used for these calculations is available from VXCERN::WJS.
} 
photons back-to-back to the collinear fermion-antifermion pair. For the case of $f=q$, an additional separation cut between the $q$ and $\bar{q}$ would separate the events into 1-jet $+n \gamma$ and 2 -jets $+n \gamma$ classes. We will quantify this below.

Fig. 1 shows the decay fractions for a muon-pair final state,

$$
R_{n}\left(y_{\mathrm{cut}}\right)=\frac{\Gamma\left(Z \rightarrow \mu^{+} \mu^{-}+n \gamma\right)}{\Gamma\left(Z \rightarrow \mu^{+} \mu^{-}\right)}
$$

as a function of $y_{\text {cut }}$ for $n \leq 4$. The values $\alpha=1 / 128, M_{Z}=91.175 \mathrm{GeV}$ and $\sin ^{2} \theta_{W}=0.230$ are assumed. For $n=1$ the calculation can in fact be performed analytically:

$$
\begin{aligned}
R_{1}(y)= & \frac{\alpha}{\pi}\left(\log ^{2} y+\frac{1}{2}(1-y)(y-3) \log \left(\frac{1-y}{y}\right)\right. \\
& \left.+\frac{5}{4}(1-2 y)+2 \operatorname{Li}_{2}(y)-\frac{\pi^{2}}{6}\right) .
\end{aligned}
$$

As expected, the rates decrease rapidly for increasing numbers of photons. Away from the edges of phase space, for example at $y_{\text {cut }}=0.01$, the rates fall roughly geometrically, each additional photon reducing the decay rate by a factor of about 100 , i.e. $\mathcal{O}(1 / \alpha)$. At larger $y_{\text {cut }}$, the phase space constraints are more severe for the multiphoton rates.

In the limit of small $y_{\text {cut }}$, the soft-photon region dominates the $R_{n}$ fractions, and it is a straightforward exercise to show - using for example the techniques of reference [9] - that the leading logarithmic behaviour is

$$
R_{n}\left(y_{\text {cut }}\right) \sim \frac{1}{n !}\left(\frac{\alpha}{\pi} \log ^{2} y_{\text {cut }}\right)^{n} .
$$

As stressed earlier, at large $y_{\text {cut }}$ only an exact matrix element calculation makes sense. We can illustrate this by repeating the calculation using the soft-photon matrix element approximation:

$$
\left|\mathcal{M}_{n}\right|^{2} \simeq\left|\mathcal{M}_{0}\right|^{2} \frac{1}{n !} \prod_{i=1}^{i=n} e_{f}^{2} \frac{2 p_{3} \cdot p_{4}}{p_{3} \cdot k_{i} p_{4} \cdot k_{i}}
$$

Table 1 shows the ratio of the approximate to exact photon fractions obtained in this way, for different $y_{\text {cut }}$ values. We see that, as expected, the approximation fails at large $y_{\text {cut }}$.

Fig. 2 shows the photon fractions calculated for hadronic final states, i.e. for five flavours of (massless) quarks. The full curve is for no separation cut between the quarks (one-jet + two-jet configurations), while the dashed curve shows the effect of imposing the additional cut $\left(p_{3}+p_{4}\right)^{2}>y_{\text {cut }} M_{Z}^{2}$ (two-jet configuration only). Not surprisingly, the difference is largest at large $y_{\text {cut }}$ - at small $y_{\text {cut }}$ the emission 


\begin{tabular}{|c|c|c|c|c|}
\hline \multirow{2}{*}{$\mathrm{n}$} & \multicolumn{4}{|c|}{$y_{\text {cut }}$} \\
\cline { 2 - 5 } & 0.001 & 0.01 & 0.1 & 0.3 \\
\hline 1 & 0.79 & 0.68 & 0.43 & 0.13 \\
2 & 0.59 & 0.49 & 0.16 & - \\
3 & 0.52 & 0.42 & - & - \\
\hline
\end{tabular}

Table 1: Ratios of approximate (soft-photon) to exact decay rates for different $y_{\text {cut }}$ and different photon multiplicities $(n)$.

of soft photons leads naturally to a two-jet configuration. Note also that because of the factor 4 difference in electric charge squared, the multiphoton final states are dominated by the $u$ and $c$ quarks. Unlike Fig.1, the results for the hadronic widths in Fig.2 are subject to higher order perturbative QCD corrections, and care must be taken in the definition of 'isolated' photons. We would expect the QCD corrections to be comparable in size to the next-to-leading order corrections to multijet final states. The $\mathcal{O}\left(\alpha_{s}\right)$ corrections to the one-photon final state have recently been calculated in reference [10].

Finally, we note that the matrix elements for the decays $Z \rightarrow q \bar{q} g+n \gamma$, from which one could for example calculate the decay into a 'three jet + isolated photons' final state, can be trivially obtained from the $q \bar{q}+(n+1) \gamma$ matrix element by adjusting the coupling constants, $e^{2(n+1)} \rightarrow C_{F} g_{s}^{2} e^{2 n}$, and the symmetry factor, $1 /(n+1) ! \rightarrow 1 / n !$.

\section{Acknowledgements}

Useful discussions with Vincenzo Innocente, Ronald Kleiss, David Stickland and Bolek Wyslouch are gratefully acknowledged. I would like to thank the Theory Division at CERN for jts kind hospitality. 


\section{References}

[1] See for example: D.R. Yennie, S.C. Frautschi and H. Suura, Ann. Phys. 13 (1691) 379; R. Kleiss et al., ' $Z$ Physics at LEP1', CERN Yellow Report 89-08 (1989), Vol.3, page 1; S. Jadach, B.F.L. Ward and Z. Wass, preprint CERNTH.5994/91 (1991); R. Kleiss, preprint CERN-TH.6155 (1991), and references therein.

[2] R. Kleiss and W.J. Stirling, Nucl. Phys. B262 (1985) 235.

[3] J.F. Gunion and Z. Kunszt, Phys. Lett. 161B (1985) 333.

[4] R. Kleiss and W.J. Stirling, Phys. Lett. 179B (1986) 159.

[5] CALKUL collaboration, F.A. Berends et al., Nucl. Phys. B264 (1986) 243.

[6] S.D. Ellis, R. Kleiss and W.J. Stirling, Comp. Phys. Comm. 40 (1986) 359.

[7] We are grateful to Z. Kunszt for providing the computer code.

[8] JADE collaboration: S. Bethke et al., Phys. Lett. 213B (1988) 235.

[9] N. Brown and W.J. Stirling, Phys. Lett. 252B (1990) 657.

[10] G. Kramer and B. Lampe, preprint DESY-91-078 (1991).

\section{Figure Captions}

[1] The ratio $R_{n}=\Gamma\left(Z \rightarrow \mu^{+} \mu^{-}+n \gamma\right) / \Gamma\left(Z \rightarrow \mu^{+} \mu^{-}\right)$as a function of the invariant mass cut between the muons and photons.

[2] As for Fig.1, but for the sum of five massless quark flavours $q=u, d, s, c, b$. The dashed lines show the decay rates when an additional invariant mass cut is imposed between the quarks. 


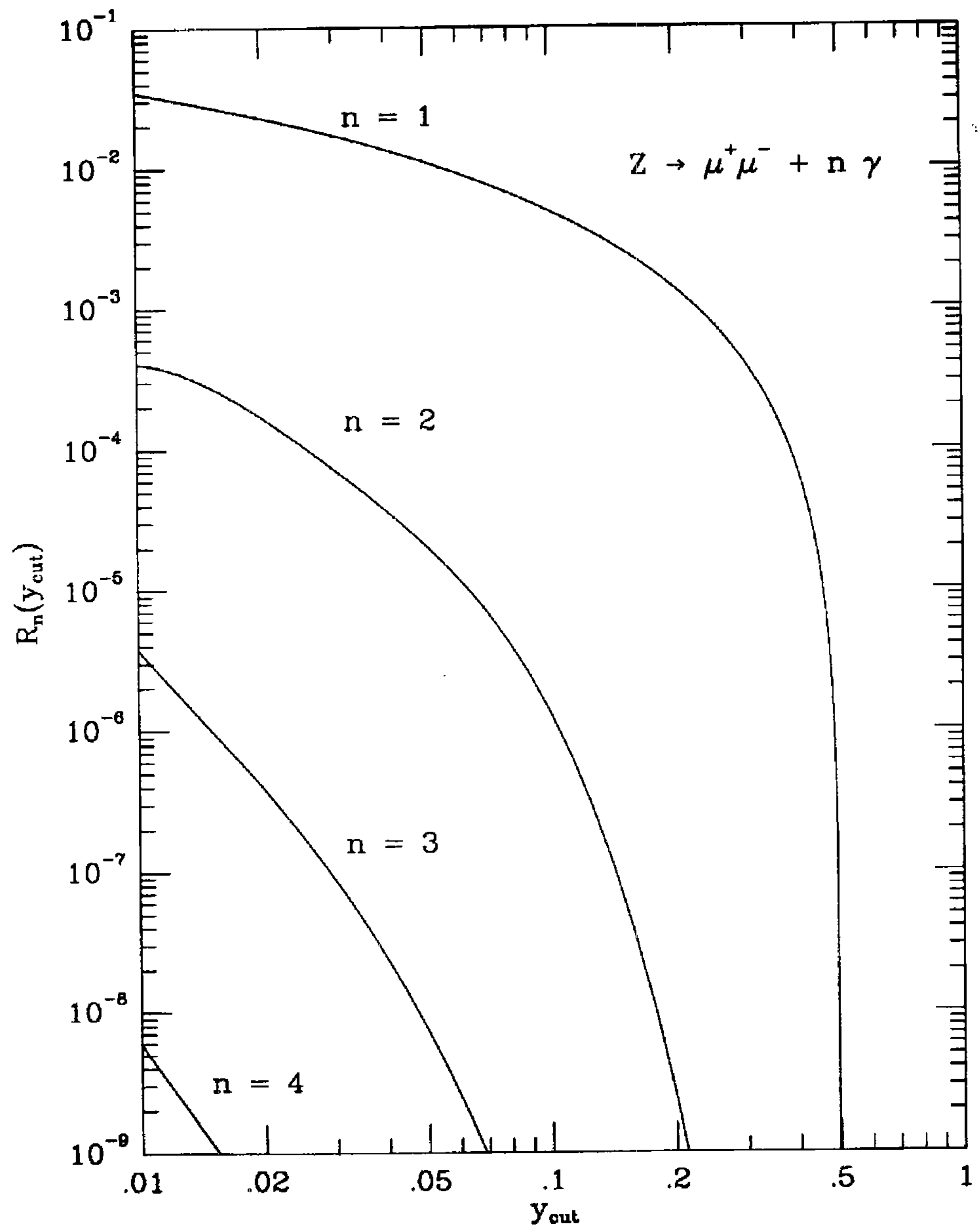

Fig , 1 


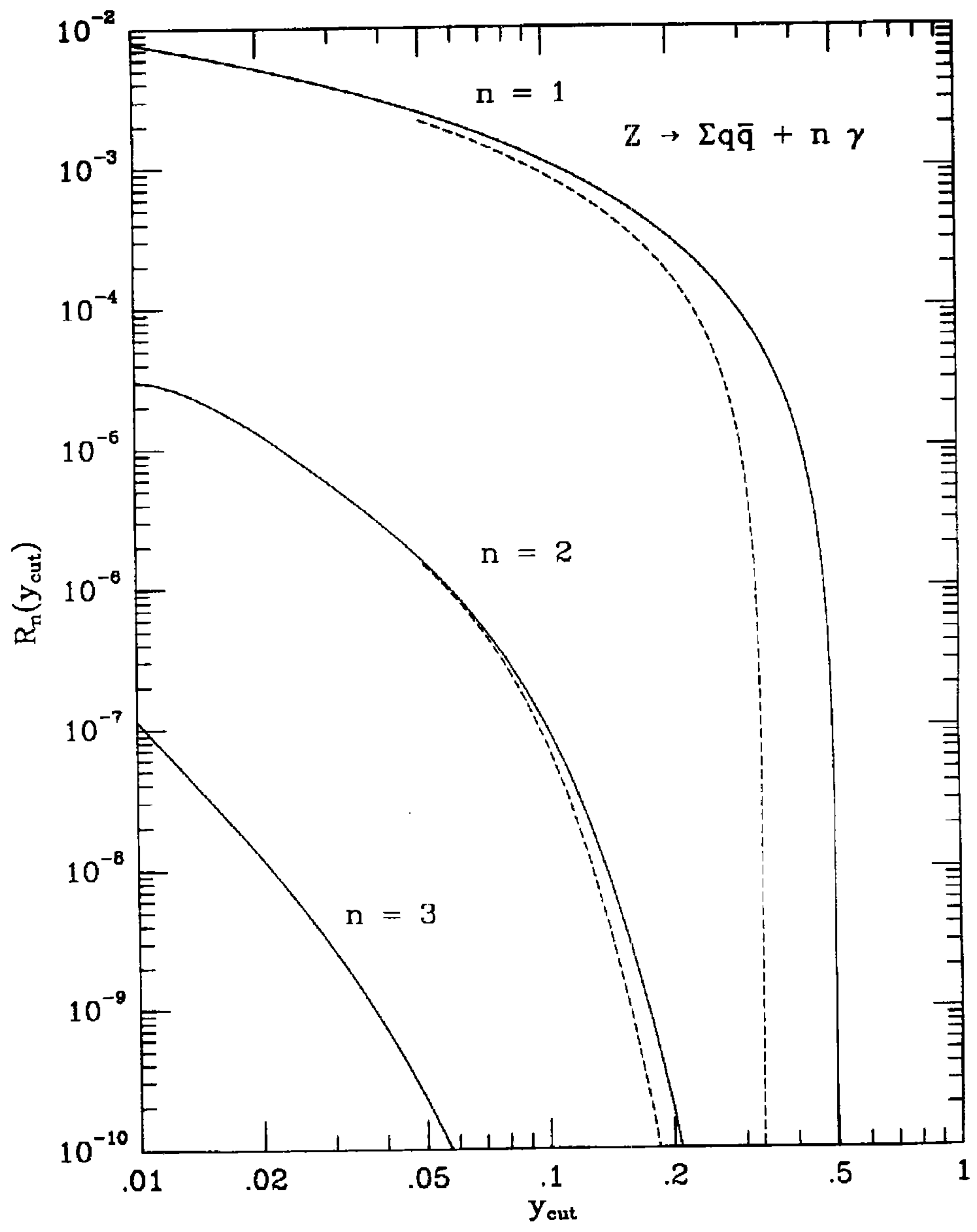

Fig. 2 